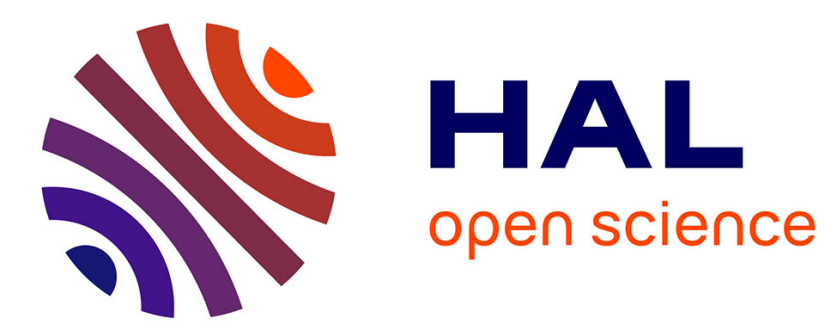

\title{
Étude de la conception d'une séquence de formation dans une filière secondaire agricole
}

\author{
Claire Masson, Laurent Veillard, Paul Olry
}

\section{To cite this version:}

Claire Masson, Laurent Veillard, Paul Olry. Étude de la conception d'une séquence de formation dans une filière secondaire agricole. Spirale - Revue de Recherches en Éducation , 2016, 59, pp.9-22. hal-02081068

\section{HAL Id: hal-02081068 \\ https://hal.science/hal-02081068}

Submitted on 12 Apr 2019

HAL is a multi-disciplinary open access archive for the deposit and dissemination of scientific research documents, whether they are published or not. The documents may come from teaching and research institutions in France or abroad, or from public or private research centers.
L'archive ouverte pluridisciplinaire HAL, est destinée au dépôt et à la diffusion de documents scientifiques de niveau recherche, publiés ou non, émanant des établissements d'enseignement et de recherche français ou étrangers, des laboratoires publics ou privés.

\section{(ㅇ)(1) $\$$}

Distributed under a Creative Commons Attribution - NonCommercial - NoDerivatives| 4.0 


\title{
ÉTUDE DE LA CONCEPTION D'UNE SÉQUENCE DE FORMATION DANS UNE FILIÈRE SECONDAIRE AGRICOLE
}

\begin{abstract}
Résumé : L'étude proposée porte sur la conception et la mise en place d'une séquence de formation au sein d'une filière professionnelle secondaire agricole. L'analyse est menée à l'aide du concept de transposition didactique interne. La méthodologie mobilisée est de type ethnographique, avec une enquête déployée sur le temps long ( 3 ans) et une combinaison de différents modes de production de données (observations, entretiens, enregistrements audio-vidéo, analyse de documents). Les analyses mettent en particulier en évidence la diversité des références à l'origine de ce processus transpositif et des opérations de transposition complexes qui dépendent pour une part importante de l'organisation sociotechnique d'un établissement, elle-même fruit d'une réflexion et de choix en partie didactiques, effectués parfois plusieurs années plus tôt.
\end{abstract}

Mots-clefs : transposition didactique interne, ateliers d'école, enseignement agricole, formation professionnelle initiale.

\section{INTRODUCTION}

L'enseignement professionnel agricole secondaire en France s'est historiquement constitué sous la forme de fermes écoles et, plus largement par la suite, d'établissements de formation qui sont aussi des unités de production (El Maaty, 2007 ; Pelpel \& Troger, 1993). Les raisons de ce choix, assez différent de celui fait dans la plupart des autres domaines de formation où les établissements n'ont qu'une finalité formative et éducative (à quelques autres exceptions près comme le domaine de l'hôtellerie restauration), tiennent à la fois à des considérations pédagogiques, des contraintes techniques, des opportunités économiques, des raisons éthiques et un projet de transformation de pratiques productives existantes.

En premier lieu, il s'agissait de proposer des cursus incluant des fortes dimensions d'apprentissage pratique dans un champ professionnel qui nécessite de manipuler du vivant (animaux, plantes, matières organiques) pour produire. La raison pour laquelle il était difficile de se limiter à des établissements uniquement formatifs avec des productions occasionnelles des élèves qui sont généralement jetées, vient d'abord d'une contrainte technique forte : les animaux, les plantes et plus largement la matière organique requièrent un soin constant qui ne peut s'arrêter lorsque les élèves sont en cours théoriques, en vacances ou en stage. Il est donc nécessaire qu'un personnel compétent soit là en permanence pour ces opérations d'élevage, de culture, de transformation et de stockage. De plus, l'idée de jeter, à l'instar des formations industrielles, les productions réalisées au sein de ces établissements heurtent tant le bon sens économique (pourquoi ne pas les vendre et assurer des rentrées d'argent à l'établissement) que des valeurs plus éthiques de 
respect du vivant et de refus du gaspillage de produits alimentaires. Enfin, il faut encore ajouter à tout cela, la volonté de faire de ces établissements des outils de transformation et de modernisation des pratiques agricoles, ce qui cadrait mal avec un modèle de type « apprentissage » où les gestes pratiques auraient été appris par des stages réguliers dans des fermes traditionnelles (cf. El Maaty, 2007).

Mais comment ces établissements peuvent être caractérisés aujourd'hui sur le plan des situations et des pratiques d'enseignement? Il n'existe pas beaucoup d'études sur ce sujet. Celles dont on dispose laissent entrevoir des dispositifs et des pratiques variables d'un établissement et d'un enseignant à un autre, au sein d'environnements organisationnels complexes et relativement contraignants (Gillet, Veyrac \& Fraisse, 2013 ; Lipp \& Ria, 2012 ; Métral, 2016). Dans cet article, nous proposons de rendre compte d'un travail de recherche en cours dans un lycée

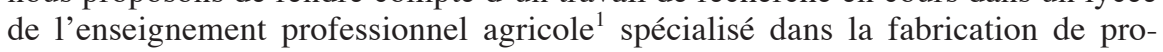
duits laitiers. L'étude présentée ici porte plus spécifiquement sur une séquence pédagogique proposée à des élèves dans une section de seconde professionnelle «Alimentation, bio-industries et laboratoire (désormais ABIL). Plus précisément, nous chercherons à répondre aux interrogations suivantes : dans ce contexte, quels sont les acteurs et les éléments organisationnels qui contribuent à configurer cette séquence pédagogique ? Quelles sont leurs modalités de contribution à ce processus de configuration ? Comment les pluralités de logiques d'actions, d'acteurs et de contraintes spécifiques de cette filière professionnelle agricole pèsentelles sur la construction d'un savoir enseignable?

Il s'agit là d'une partie d'une étude plus générale menée dans le cadre d'une thèse qui vise à analyser les caractéristiques des situations de formation et d'apprentissage mises en place dans ces ateliers et de comprendre leurs effets sur les activités et les apprentissages effectifs des élèves. Nous proposons d'aborder ces questions à l'aide du concept de transposition didactique interne. Dans la suite de cet article, nous explicitons d'abord ce choix théorique, avant de rendre compte de la démarche ethnographique mise en œuvre. Puis nous présentons et discutons les résultats obtenus.

\section{APPROCHE THÉORIQUE}

Le concept de transposition didactique a été introduit par le sociologue $\mathrm{M}$. Verret (1975) afin de rendre compte des transformations réalisées sur des savoirs lorsque l'on vise leur transmission dans une institution éducative (pour ce qui le concerne, c'était à l'université). De façon très synthétique, la thèse développée est qu'une telle visée passe nécessairement par un apprêt de ces savoirs dont Verret a proposé de rendre compte par différents types d'opérations (dépersonnalisation, désyncrétisation, programmation, publicité et définition des modes d'évaluation). Ces opérations sont rendues nécessaires à la fois par les contraintes propres à tout acte de transmission (nécessité de sélectionner certains savoirs, de planifier leur acquisition dans le temps et de vérifier celle-ci), mais aussi par l'organisation particulière des institutions éducatives, caractérisée notamment par une division du travail formatif entre différentes disciplines d'études (désyncrétisation), ou encore la nécessité de communiquer au public les objectifs d'enseignement (publicité).

La popularité de ce concept en didactique doit beaucoup à sa reprise par Chevallard (1985/1991) pour analyser l'origine des savoirs enseignés en mathé-

${ }^{1}$ Il s'agit de l'École Nationale d'Industrie Laitière et des BIOtechnologies (ENILBIO) de Poligny. 
matiques et les transformations qu'ils subissent avant de pénétrer dans les classes. L'idée force qui ressort du travail de Chevallard est que les savoirs enseignés, s'ils tirent leur légitimité de notions et de méthodes issues des mathématiques savantes, en sont assez éloignés du fait des contraintes propres à leur enseignement et au fonctionnement des institutions éducatives. Les différentes opérations élaborées par Verret (désyncrétisation, etc.) sont reprises pour rendre compte de ces transformations, dans le cadre plus restreint d'un seul domaine de savoir. En s'intéressant de près aux différents acteurs et institutions (qualifiés dans leur globalité de noosphère) qui interviennent dans la construction d'un programme d'enseignement, Chevallard montre que ceux-ci relèvent assez largement d'une construction sociale. Il propose aussi de considérer que la transposition ne s'arrête pas au texte des savoirs à enseigner (Programme). Il ne s'agit que de la composante externe d'un processus qui se poursuit à l'intérieur des institutions éducatives, par le travail des enseignants qui interprètent ces textes prescriptifs et font certains choix aboutissant aux situations concrètes d'apprentissage au contact des élèves. Il s'agit d'une seconde composante que Chevallard nomme transposition didactique interne, qui marque la transformation du savoir à enseigner en savoir enseigné.

Par la suite, le concept de transposition a largement diffusé dans d'autres didactiques disciplinaires, tout en faisant fortement débat sur certains points. En particulier, l'idée d'une origine et d'une légitimité exclusivement (ou principalement) savantes des savoirs enseignés dans les institutions éducatives s'est assez vite heurtée à des oppositions importantes dans certaines disciplines comme le français, la technologie, ou encore dans le cas des enseignements en formation professionnelle.

Le concept de pratique sociale de référence introduit par Martinand (1986) a nourri ce débat, même si la notion n'avait pas été pensée initialement comme une critique ou même un enrichissement de la théorie de la transposition didactique. Cette notion a ouvert sur des questions telles que : qu'est-ce qui est transposé ? Uniquement des savoirs ou bien également les pratiques sociales qui les mobilisent et/ou les produisent, voire les outils liés à ces pratiques (Perrenoud, 1998) ? Peut-on d'ailleurs considérer qu'il y a toujours des savoirs dans toutes les pratiques humaines, en envisageant par exemple des savoirs experts (ceux jugés comme essentiels par des artisans charpentiers et leurs institutions) à côté des savoirs savants (Joshua, 1997) ? Peut-on envisager une pluralité de références dans certains domaines d'enseignement, y compris certains savoirs et pratiques qui seraient propres aux espaces scolaires (Durey, 2002) ? Dans le cas des formations professionnelles initiales, Raisky (1996) souligne que ce type de formation vise non pas tant l'acquisition de savoirs que la maîtrise des situations de travail liées à un type de métier ou de fonction. Une telle maîtrise nécessite de plus en plus des compétences aux composantes multiples (scientifiques, techniques, pratiques, relationnelles, etc.), étant donné les évolutions des sphères professionnelles. Il est donc tout à fait logique que ces filières de formation combinent des enseignements de savoirs renvoyant à plusieurs sphères de pratique sociales (savantes, professionnelles, etc.).

Nous n'avons pas la place dans le cadre de cet article pour rendre compte des nombreux débats autour du concept de transposition didactique et de la possibilité de sa généralisation à tous les domaines d'enseignement et de formation (Arsac, Chevallard, Martinand, \& Tiberghien, 1994 ; Caillot, 1996 ; Mercier, 2002). Nous considérons pour notre part qu'il est possible et intéressant de le mobiliser pour mieux comprendre la genèse des situations de formation pratique dans un établissement tel qu'un lycée professionnel. Dans notre cas, c'est la transposi- 
tion didactique interne qui nous intéresse plus particulièrement, c'est-à-dire les différentes opérations réalisées dans un établissement aux caractéristiques organisationnelles, spatiales, techniques et temporelles particulières, pour concevoir une séquence d'enseignement dans les ateliers. Précisons que, de notre point de vue, ces opérations de transposition ne portent pas a priori uniquement sur des savoirs (en l'occurrence ceux qui figurent dans les référentiels nationaux). Elles peuvent aussi concerner des tâches, des formes d'organisation, des rôles, des machines ou des outils professionnels qui sont modifiés, adaptés au niveau de l'établissement pour organiser des situations et des activités des élèves censées permettre certains apprentissages.

On aurait pu aborder la problématique qui nous intéresse (conception d'une séquence de formation) sous l'angle de la pratique ou de l'activité enseignante, comme les travaux mentionnés en introduction de cet article ont choisi de le faire. Nous avons préféré l'envisager du point de la transposition interne, car il nous semble que cette notion permet de se situer à un autre niveau que celui des pratiques individuelles des enseignants et ainsi d'englober un ensemble plus large d'acteurs, d'éléments organisationnels et matériels jouant un rôle dans la constitution des situations d'apprentissage pratique des élèves. De la même façon que la partie externe de la transposition didactique (aboutissant au texte des savoirs à enseigner) met en jeu plusieurs acteurs et institutions (regroupés sous le terme de noosphère par Chevallard), notre hypothèse est que sa partie interne ne dépend pas que de l'action d'un ou de quelques enseignants. Ceux-ci doivent faire localement avec un ensemble d'opportunités et de contraintes qui sont liées à l'organisation sociale et technique de l'institution. Dans une telle perspective, la transposition didactique interne doit donc être replacée dans le contexte local de cette institution, en tant que processus social complexe et situé. C'est une partie de cette complexité que l'on voudrait illustrer empiriquement dans cet article.

\section{MÉTHODOLOGIE}

Pour mener notre étude, nous avons adopté une méthode d'inspiration ethnographique qui se caractérise par une enquête au long cours (trois ans) dans l'établissement et son environnement. Une telle approche se justifie par le fait que l'on s'intéresse à un processus social complexe tel que décrit ci-dessus dans la partie théorique.

Comprendre un tel processus, renvoyant à différents moments, lieux, acteurs et éléments de l'organisation, requiert de pouvoir en reconstituer les tenants et les aboutissants au moyen d'une approche que l'on peut qualifier de multisituée (Marcus, 1995). En d'autres termes, cela signifie que, si notre objet d'étude est bien une certaine séquence d'apprentissage locale, nous l'envisageons comme le résultat d'un processus de transposition qui nécessite, pour pouvoir le comprendre, d'enquêter dans d'autres lieux, d'autres temps et à d'autres niveaux que dans les seules salles de cours et les ateliers où se déroulent la séquence. Par exemple, on verra l'importance de questionner quelque peu l'histoire de cet établissement et de son environnement institutionnel et économique qui l'ont conduit à des choix de missions multiples qui ne sont pas sans conséquence sur les possibilités de mise en place de la séquence qui nous intéresse.

Une enquête ethnographique de ce type nécessite de combiner différents modes de production de données (Olivier de Sardan, 1995).

1) Un recueil de documents de deux types : certains propres à l'établissement, soit à usage externe (sites internet, documents de communication vers les 
élèves ou le milieu professionnel...), soit à usage interne (plans des bâtiments, schémas des installations, règlements de la halle, projet pédagogique, fiche de fabrication, documents pédagogiques des enseignants, etc.); d'autres produits par des institutions externes, comme des textes issus de la branche professionnelle (institut technique et professionnel du Comté) ou encore par le ministère de l'agriculture au niveau national (ex : référentiels de la seconde ABIL, des brevets d'études professionnelles agricoles (BEPA) et baccalauréats professionnels correspondants). L'analyse de ces documents nous a servi à comprendre les différentes missions que s'est fixé l'établissement et la façon dont il s'est organisé historiquement pour pouvoir y répondre.

2) Des entretiens enregistrés avec plusieurs enseignants et formateurs, complétés par des échanges plus informels avec différents acteurs de l'établissement (directeur, salariés). Ces entretiens nous ont permis de compléter l'analyse de l'organisation de l'établissement, mais aussi de documenter les opportunités et les contraintes identifiées par les concepteurs de la séquence et la façon dont ils ont décidé de la mettre en place concrètement en réalisant certains choix didactiques.

3) Enfin, nous avons aussi mené des observations sur site, à l'aide d'un carnet d'observations (notes, schémas, photos) et en ayant recours à des enregistrements audio-vidéographiques (plus d'une centaine d'heures), en particulier pour garder des traces très fines de la façon dont la séquence a été conçue lors de réunions, puis s'est déroulée dans les ateliers. Toutefois ces données d'observation ne seront pas utilisées ici pour analyser ce déroulement de la séquence, mais pour compléter l'analyse de l'organisation de l'établissement (configuration concrète des salles, types de machine présents, etc.) et celle de la conception de la séquence.

\section{RÉSULTATS}

Pour présenter les résultats de notre analyse, nous avons choisi de procéder de façon descendante afin de faciliter la compréhension du lecteur. Dans un premier temps, nous rendrons compte des grandes missions de l'établissement et de l'organisation générale mise en place dans son contexte régional spécifique pour assurer ces missions, à la fois sur le plan de la division du travail et de la configuration spatiale. Puis, nous nous intéresserons plus précisément aux ateliers, où la séquence étudiée s'est déroulée en grande partie, pour rendre compte de la façon dont ils sont organisés, dans une recherche d'équilibre entre des buts productifs et formatifs. Enfin, dans une dernière partie, nous nous situerons au niveau de la séquence, pour l'analyser du point de vue des choix de ses concepteurs, en regard des contraintes et des opportunités auxquelles ils ont dû faire face compte-tenu de l'organisation du travail au sein de l'établissement et de la configuration technique des ateliers.

\section{Un établissement doté de plusieurs missions}

L'ENILBIO de Poligny est un établissement public de formation dépendant du ministère de l'agriculture, dans le domaine de l'agroalimentaire. Créé en 1889 , en tant qu'école professionnelle, l'établissement a pour mission principale de former le personnel des entreprises locales dans le domaine de la transformation laitière. Il fait partie d'un tissu professionnel très organisé autour de la filière du Comté (Jura), offrant une ressource importante pour son développement sur les 
plans économique et technique. À ce titre l'ENILBIO assure, outre sa mission première de formation ${ }^{2}$, d'autres missions synthétisées dans ce tableau.

\begin{tabular}{|c|c|c|}
\hline \multicolumn{3}{|c|}{$\begin{array}{l}\text { Formations } \\
\text { Préparer des jeunes et des adultes aux métiers de la transformation } \\
\text { et du contrôle des produits agro-alimentaires et laitiers. }\end{array}$} \\
\hline $\begin{array}{l}\text { Type de formation : initiale (du } \\
\text { certificat d'aptitude profession- } \\
\text { nelle (CAP) au brevet de techni- } \\
\text { cien supérieur (BTS)) } \\
\text { Institution : Lycée } \\
\text { Lieux : salles de classe, halle de } \\
\text { génie alimentaire, lieux de } \\
\text { stages (entreprises) } \\
\text { Acteurs : enseignants, forma- } \\
\text { teurs-techniciens, tuteurs de } \\
\text { stage }\end{array}$ & $\begin{array}{l}\text { Type de formation : apprentis- } \\
\text { sage } \\
\text { Institution : Centre de Forma- } \\
\text { tion d'Apprentis (CFA) } \\
\text { Lieux : salles de classe, halle de } \\
\text { génie alimentaire, lieux } \\
\text { d'alternance (entreprises) } \\
\text { Acteurs : enseignants, forma- } \\
\text { teurs-techniciens, tuteurs }\end{array}$ & $\begin{array}{l}\text { Type de formation : continue } \\
\text { courte/longue ; qualifiante/ certi- } \\
\text { fiante } \\
\text { Institution : Centre de Formation } \\
\text { Professionnelle et de Promotion } \\
\text { Agricole (CFPPA) } \\
\text { Lieux : salles de classe, halle de } \\
\text { génie alimentaire, stages de } \\
\text { formation à distance. } \\
\text { Acteurs : enseignants, forma- } \\
\text { teurs-techniciens, tuteurs }\end{array}$ \\
\hline \multicolumn{3}{|c|}{ Autres missions } \\
\hline $\begin{array}{l}\text { Production et commercialisa- } \\
\text { tion des produits agro- } \\
\text { alimentaires } \\
\text { Lieux }: \text { ateliers, magasin } \\
\text { Acteurs : formateurs-techniciens }\end{array}$ & $\begin{array}{l}\text { Conseil et service d'analyse } \\
\text { aux entreprises de la région : } \\
\text { technologies de production, } \\
\text { qualité, sécurité, analyses physi- } \\
\text { co-chimiques et bactériolo- } \\
\text { giques. } \\
\text { Lieux : ateliers, laboratoires } \\
\text { Acteurs : formateurs- } \\
\text { techniciens, enseignants, chefs } \\
\text { de projet }\end{array}$ & $\begin{array}{l}\text { Expérimentation, recherche fi- } \\
\text { nalisée ou appliquée, majoritai- } \\
\text { rement dans le domaine des } \\
\text { technologies laitières, en parte- } \\
\text { nariat avec des entreprises du } \\
\text { secteur et les établissements su- } \\
\text { périeurs } \\
\text { Lieux : laboratoires, ateliers } \\
\text { Acteurs : formateurs- } \\
\text { techniciens, enseignants, chefs } \\
\text { de projet }\end{array}$ \\
\hline
\end{tabular}

Tableau 1 : les différentes missions de l'établissement

Le tableau 1 fait apparaître que plusieurs lieux de l'établissement sont partagés pour assurer les différentes missions. De même, on constate que les différents acteurs mentionnés contribuent à plusieurs de ces missions et qu'ils ne sont donc pas centrés sur une seule d'entre elle. Tout cela signifie donc que l'organisation technique et humaine doit être suffisamment polyvalente, ou pour le dire autrement très « hybride» pour assurer des actions aussi diverses que de la formation initiale longue de jeunes, de la formation continue courte ou longue d'adultes, de la production et de la vente quotidiennes, du conseil, de l'expertise et des projets d'expérimentations avec des entreprises. L'établissement dispose pour cela d'une halle de génie alimentaire d'environ $4000 \mathrm{~m}^{2}$ relativement récente (moins de 10 ans).

Cette halle peut être décomposée en quatre zones d'activités (cf. figure 1).

- Une zone de production et de formation en agro-alimentaire au rez-dechaussée, comprenant trois ateliers de fabrication (produits frais, fromages à pâtes molles, fromages à pâtes pressées), une salle de lavage, une zone de conditionne-

${ }^{2}$ Article R811-9 du code rural français : « [...] L'atelier technologique est une unité de traitement, de transformation et de vente de produits obtenus à partir de matières premières agricoles introduites ou produites sur l'exploitation ou une unité de services vendus à des particuliers ou à des collectivités. Leur orientation, leur conduite et leur gestion, qui se réfèrent aux usages et pratiques commerciales des professions concernées, sont utilisées comme moyens de formation, d'expérimentation, de démonstration et de développement. » 
ment et d'expédition, une salle d'affinage, une cave (en pointillés sur la figure 1) et des salles de cours attenantes.

- Une zone de visite au $1^{\text {er }}$ étage, ouverte au public, matérialisée par une galerie vitrée surplombant les salles de génie alimentaire et de transformation fromagère sur toute la longueur du bâtiment. Elle débouche à l'entrée du magasin de vente directe, où les visiteurs peuvent acheter les produits.

- Une zone (en traits gras) comprenant deux laboratoires où sont réalisés certains travaux pratiques (TP) relatifs à des activités de bio-transformation mais aussi des analyses (qualité, hygiène) et des expérimentations en recherche et développement et un certain nombre de services rendus aux entreprises.

- Une zone comprenant des salles de cours et de TP de sciences qui peuvent aussi bien servir aux cours qu'à des réunions avec des partenaires externes

- Une zone comprenant les bureaux des enseignants, des formateurs et autres salariés de l'établissement.
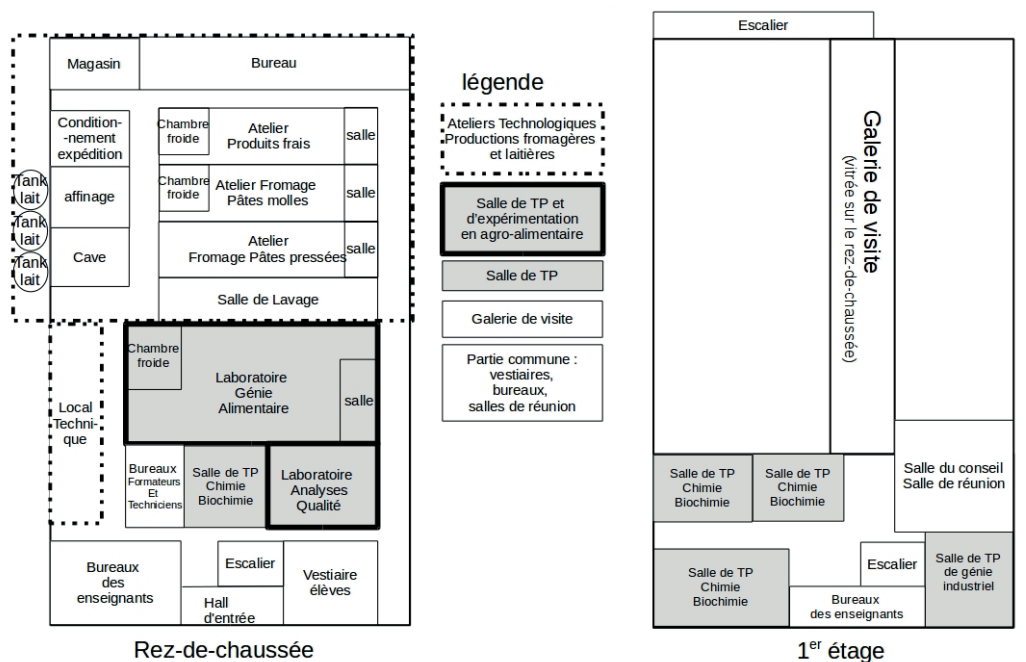

Figure 1 : plan de la halle de génie alimentaire

Dans le cadre réduit de cet article, il n'est pas possible d'étudier tous ces espaces. Nous allons nous focaliser sur les ateliers technologiques dans la mesure où ils constituent un lieu privilégié pour la séquence qui nous intéresse.

Des ateliers pour produire ...

Les trois ateliers reprennent chacun une organisation technique de transformation laitière par bien des points très semblable à ce qui peut exister dans les fromageries de la région, les fruitières. On y trouve en particulier : des tanks de stockage, une tuyauterie à commande numérique de gestion du lait et des fluides, un nettoyage en place ou encore une «marche en avant ${ }^{3} »$. Chaque atelier est spécialisé dans la fabrication d'un type de produit, et suit à ce titre des règles de fa-

${ }^{3} \mathrm{La}$ «marche en avant » est une procédure pour garantir l'hygiène et la sécurité alimentaire avec pour principe de base que les produits d'une étape du process de fabrication ne doivent pas croiser le chemin des produits d'autres étapes, notamment les déchets, pour éviter la contamination. 
brication spécifiques, avec des matériels à l'avenant. Ainsi l'atelier « Produits Frais » est de type industriel avec un nombre important d'automatismes. L'atelier «Pâte pressée » suit les règles de fabrication en vigueur de l'Appelation d'Origine Protégée (AOP) Comté qui impose par exemple de travailler, en lait cru et dans les 24 heures suivant la traite, un lait issu d'une collecte limitée à un cercle de $25 \mathrm{~km}$ autour du lieu de transformation. L'organisation de l'atelier «Pâte molle » est identique à celle que l'on retrouve dans les fruitières de la région de Poligny. Un enjeu financier très important est engagé chaque jour quand, par exemple, il s'agit de transformer 1200 litres de lait cru de qualité AOP, ce qui correspond à 3 tomes de $40 \mathrm{~kg}$ de comté. De ce fait, les erreurs sont peu permises. Les produits fabriqués sont vendus pour dégager des revenus nécessaires au fonctionnement de ce centre au sein de l'établissement (y compris les salaires de certains personnels), par un réseau de commercialisation : soit par le biais du magasin sur place, soit par la vente à des clients dans la région (grande distribution, etc.).

Ces lignes de fabrication (et plus généralement l'ensemble du bâtiment) ont été construites dans le strict respect de la réglementation pour l'hygiène et la sécurité alimentaire, très développée dans ce secteur d'activité. Cette réglementation s'applique à la fois à l'organisation matérielle des lignes mais aussi aux pratiques et processus de fabrication qui sont mis en œuvre. Par exemple, tous les acteurs, incluant bien évidemment les élèves doivent observer le port des tenues réglementaires (bottes, vêtements, charlottes, protections auditives) et des règles d'hygiène. Les installations doivent également être méticuleusement nettoyées après chaque cycle de fabrication

Sur le plan de l'organisation du travail, les ateliers de transformation fonctionnent toute l'année, de la collecte du lait à la commercialisation, six jours sur sept, y compris pendant les périodes de congés scolaires. Un directeur (fonctionnaire), quatre salariés et cinq formateurs-techniciens (recrutés pour leur expertise technique dans la production laitière, titulaires en général d'un BTS) travaillent à temps complet dans ces lieux. Les quatre salariés gèrent les commandes de matières premières, les soins d'affinage, les préparations de commande et les livraisons et les formateurs-techniciens assurent la responsabilité du laboratoire qualité et d'une ligne de production.

... mais aussi pensés pour la formation ...

Cependant, si ces ateliers constituent des dispositifs de production à fort enjeu économique, certaines de leurs caractéristiques en font aussi des lieux différents d'une situation productive ordinaire. Au moment de leur conception, des choix d'organisation de l'espace et de dispositifs techniques ont été faits dans l'optique de permettre la familiarisation et l'apprentissage de certaines activités productives. En particulier, les trois ateliers se composent d'une collection de matériels et de types de fabrication permettant une mise en contact avec une diversité de produits, de dispositifs techniques et de gestes de fabrication de ce secteur productif. Ainsi, l'établissement fabrique un ou plusieurs produits par grande famille de fromages et de produits frais. Ces produits vont de l'agriculture biologique à des fromages plus industriels, commercialisés en grande distribution. Certains processus de fabrication sont repris de la grande industrie, mais d'autres sont typiques de ceux qui sont mis en œuvre dans les nombreuses petites coopératives existantes dans la région. On ne trouve nulle part dans le monde professionnel de la transformation laitière un plateau technique aussi éclectique.

Si l'on regarde de plus près le matériel en place, on s'aperçoit que certaines de leurs caractéristiques permettent de rendre visibles des gestes ou des processus 
(physico-chimiques, techniques) et de faciliter les explications. Ainsi plusieurs éléments ont été placés pour pouvoir être correctement observés depuis la galerie de visite et l'organisation de l'espace autorise la présence de plusieurs personnes autour des appareils (cf. figure 2 ci-dessous). Le choix a aussi été fait de ne pas tout automatiser, car cela aurait masqué des opérations importantes à comprendre. C'est par exemple le cas de l'opération de remplissage des pots de fromage blanc qui est très souvent automatisée dans les laiteries (cf. figure 3 ).

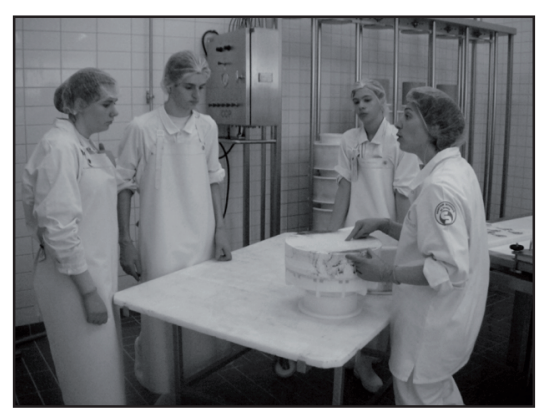

Figure 3 : le remplissage des pots de fromage blanc

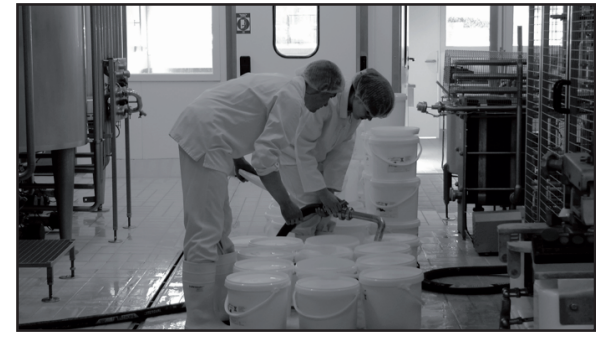

Figure 2: des espaces permettant l'échange

Par ailleurs, chaque atelier de fabrication dispose d'une salle de cours très proche des lignes de fabrication, avec des tables, un tableau, des documents accessibles concernant la fabrication. Sur le plan de la figure 1, on peut voir que chaque atelier dispose d'une telle salle attenante. Cet aménagement permet de basculer rapidement dans une configuration proche de celle d'un cours classique (et vice et versa), d'autant plus qu'il n'est pas nécessaire de changer de tenue.

...en tension entre ces deux logiques

On peut cependant noter que seul du matériel professionnel a été choisi pour les ateliers : cuves, tanks, automates, etc. Ce sont les mêmes ceux que les apprenants trouvent dans les entreprises qui les accueillent. Dans cette partie de la halle il n'existe pas de matériel didactique, comme on peut en trouver dans les laboratoires d'analyse et de génie alimentaire où se déroulent d'autres travaux pratiques, ou comme il en existe dans de nombreux lycées professionnels. Là s'arrêtent sans doute les possibilités d'aménagement didactique d'installations de ce genre qui doivent assurer une bonne productivité et s'avérer très fiables sur le long terme. Cette logique productive est aussi en adéquation avec la logique de construction de ces ateliers que l'établissement voulait le plus proche possible de situations d'entreprises.

Mais il n'y a pas que la logique productive qui contraigne l'action de formation. L'inverse est également vrai. Un exemple intéressant de ce point de vue, est le rythme hebdomadaire des prévisions de fabrication sur les lignes. Si ces prévisions varient en fonction des volumes de vente et des stocks de produits finis disponibles comme dans toute entreprise, elles dépendent aussi fortement du nombre d'apprenants présents chaque jour dans l'atelier technologique. En effet, sauf exception, les fabrications sont réalisées par ces apprenants (trois à six par atelier idéalement), encadrés par un formateur-technicien. Il peut s'agir d'étudiants en BTS, de lycéens, d'apprentis, de stagiaires en formation continue, ou encore de professionnels envoyés par leur entreprise pour des formations courtes. Un programme des matinées est établi au début de l'année, définissant pour chaque pro- 
motion les semaines de passage (de deux à six par an selon les filières) sur les ateliers. Les apprenants restent sur le même atelier pendant une semaine complète, mais doivent changer de poste pour pouvoir se former aux différentes opérations du processus de fabrication.

L'organisation de la production doit donc nécessairement intégrer cette contrainte de nature formative au même titre que celles d'ordre technique et économique. À tel point que, en fonction du nombre d'élèves ou de formés présents dans l'atelier, le volume de lait transformé peut être ajusté. Le surplus est alors revendu à la coopérative chargée du ramassage du lait. Enfin, on peut également noter que, pendant les périodes de congés scolaires, une permanence est assurée par les apprenants et les formateurs, ce qui montre cette fois, à l'inverse, comment la continuité de la logique productive exerce une forte contrainte, sur les formateurs et les formés.

Un exemple de transposition interne

en seconde professionnelle ABIL

Dans le contexte généré par cette organisation générale de l'établissement et des ateliers en particulier, nous allons maintenant étudier la façon dont une séquence de formation pratique a été conçue et mise en place dans la seconde professionnelle $\mathrm{ABIL}^{4}$.

À l'origine de la séquence, il y avait la volonté de l'équipe enseignante de mieux préparer les élèves à une épreuve du BEPA qui se passe depuis quelques années en cours de formation. Cette épreuve orale consiste à présenter des fiches d'activité rendant compte de tâches réalisées lors du stage en milieu professionnel (fin de seconde) et qui correspondent à des situations significatives répertoriées dans le référentiel du diplôme. Les enseignants avaient constaté que les élèves avaient beaucoup de mal à réussir cette épreuve sans préparation préalable. L'établissement disposant d'un volant d'heures laissées à son initiative, l'enseignante coordinatrice des secondes et un de ses collègues ont proposé d'en utiliser un certain nombre ( 35 heures) afin de mettre en place une séquence visant à préparer les élèves à réaliser ce type de fiche réflexive et à les expliquer à l'oral avant qu'ils ne partent en stage.

Au-delà cette préparation à l'épreuve du BEPA, l'équipe enseignante a rapidement entrevu l'intérêt d'une séquence de ce type pour essayer de pallier à des difficultés plus générales des jeunes lycéens. En effet, leur cursus de seconde s'avère complexe par rapport au collège dans la mesure où ils doivent être en capacité de s'adapter à des contextes d'apprentissage très différents : le lycée, l'internat, les laboratoires, les ateliers, le lieu de stage en entreprise. La création de la séquence était donc l'occasion de tenter de mettre en place un accompagnement de ce parcours de formation. Il s'agissait notamment :

- de s'assurer qu'ils disposent des connaissances techniques minimales avant qu'ils ne partent en stage en milieu professionnel ;

- de développer chez eux une forme de pré-socialisation avec ce type d'environnement de travail, qui inclut aussi la capacité à respecter concrètement certaines normes d'action et de comportement, à s'approprier des valeurs de cette culture professionnelle ;

${ }^{4}$ La seconde ABIL est une année préparatoire, préalable au choix d'une filière de baccalauréat professionnel à partir de la première : soit Bio-Industrie de Transformation, soit Laboratoire Contrôle Qualité. 
- de les préparer à l'analyse réflexive et la mise en mots des tâches réalisées en situation de travail, qui correspond non seulement aux exigences de l'épreuve du BEPA, mais aussi à des demandes de traçabilité croissantes dans les entreprises.

On constate donc que les références du processus de transposition didactique interne sont multiples : certaines émanent du système d'enseignement agricole (référentiels du BEPA et de la seconde ABIL), d'autres de l'environnement socio-économique local (attentes d'entreprise partenaires pour le stage), et d'autres enfin de l'établissement lui-même (capacités jugées nécessaires pour réussir le parcours de formation). Par ailleurs, ces références ne sont pas décrites simplement sous les termes de «connaissances » ou de « compétences ». Dans les documents étudiés (référentiel du BEPA, référentiel ABIL, etc.), ou lors des entretiens avec nos interlocuteurs (enseignants, formateurs), si ces termes apparaissent régulièrement, on en trouve aussi de nombreux autres comme «situations professionnelles significatives », « activités », « valeurs », «culture professionnelle », « normes », « capacités », etc.

Si l'on rentre maintenant un peu plus dans le détail, la séquence, réalisée en demi-classe, se décompose de la façon suivante (cf. figure 4).

Schéma temporel de la séquence étudiée pour une demi-classe
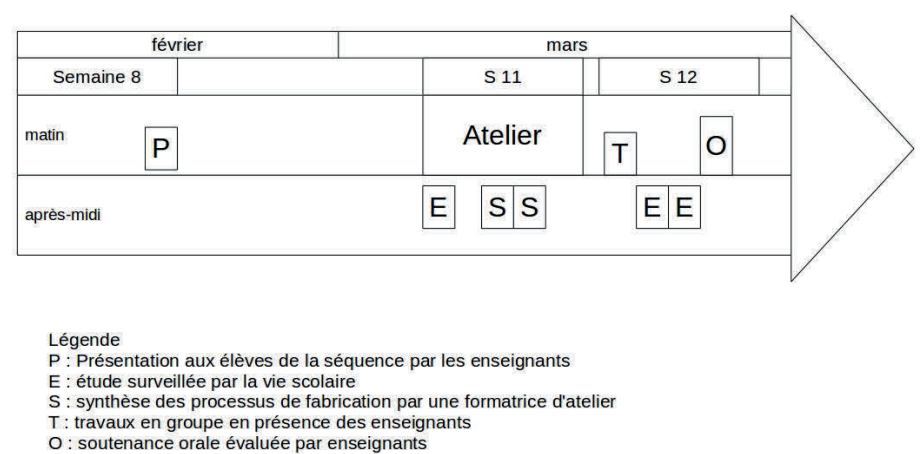

E : étude surveillée par la vie scolaire
$S$ : synthèse des processus de fabrication par une formatrice d'atelie
$T$ : travaux en groupe en présence des enseignants

$O$ : soutenance orale évaluée par enseignants

Figure 4 : vue schématique du déroulement la séquence

- 2 heures de présentation de la séquence en salle de cours, animées par les 2 enseignants concepteurs et responsables du module.

- 1 semaine complète au cours de laquelle les élèves sont par groupe de 5 ou 6 et participent tous les matins aux travaux des ateliers encadrés par les formateurs-techniciens.

- 10 heures de travaux de groupe dans une salle de cours, dont 2 heures encadrées par les 2 enseignants, le reste en étude avec des assistants d'éducation de la vie scolaire. Durant ces temps les élèves, par groupes de 2 ou 3 , élaborent deux fiches d'activités similaires à celles attendues pour l'épreuve de BEPA : une première sur une activité de production et une autre sur une activité d'analyse et contrôle. 
- 3 heures de restitution sous forme de soutenance orale devant les 2 enseignants et le demi-groupe.

Deux points apparaissent singuliers dans cette organisation. Nous allons voir qu'ils sont directement liés aux spécificités organisationnelles et techniques de l'établissement.

En premier lieu, ce ne sont pas les deux enseignants qui encadrent les élèves lors des séances pratiques en ateliers, mais les formateurs-techniciens dont on a déjà expliqué plus haut qu'ils avaient été recrutés d'abord sur leur capacité à gérer des situations productives, ils n'ont donc pas de formation à la pédagogie. D'une certaine façon, leur rôle se situe à mi-chemin entre celui d'un enseignant d'une discipline professionnelle (semblable à celui d'un professeur d'atelier dans d'autres spécialités) et celui d'un tuteur en situation de travail qui doit gérer de fortes contraintes productives. Si l'on ajoute que les séances de travail réflexif en classe sont majoritairement animées par des assistants d'éducation, ce sont au final trois types d'acteurs qui interviennent auprès des élèves. Cette répartition de l'encadrement a été rendue nécessaire par la division du travail en vigueur dans l'établissement.

En second lieu, les situations vécues et les tâches réalisées par les élèves pendant la semaine dans les ateliers dépendent de l'organisation propre de ceux-ci, avec un planning général qui, comme nous l'avons indiqué, est établi au début de l'année. Au cours de ces séances, la quinzaine d'élèves présents est divisée en trois petits groupes de quatre ou cinq. Chaque groupe se voit attribuer une opération spécifique : préparation des produits frais, des pâtes molles ou encore affinage ou conditionnement des produits. Le lendemain, les groupes changent d'opération. En plus des postes à l'atelier, les élèves se rendent aussi à tour de rôle dans les laboratoires, une matinée dans la semaine, pour y effectuer les contrôles biochimiques et chimiques nécessaires à la production. Enfin, au-delà de l'occupation des postes, il est attendu que chaque élève participe et se rende disponible en cas de nécessité, lors d'urgences ou d'étapes plus intensives du processus productif.

Les enseignants concepteurs de la séquence n'ont donc pas la main sur une planification de tâches et de situations qui préexistaient à la conception de leur séquence. Ils ont donc dû s'adapter en étalant la séquence sur une période permettant aux deux groupes de faire un passage sur un même atelier pour des activités similaires. Il arrive même que des aléas productifs (par exemple une machine capricieuse ou en panne) conduisent à ce que les élèves ne puissent réaliser certaines tâches dans la semaine. D'ailleurs, en conséquence, les animateurs des séances de travail en classe ne sont pas toujours au courant de ce qui s'est passé à l'atelier, ce qui peut conduire à des incompréhensions et des quiproquos avec les élèves, surtout lorsque ce sont des assistants d'éducation qui encadrent les séances dans la mesure où ceux-ci n'ont pas toujours les connaissances techniques pour comprendre ce que leur expliquent les élèves.

\section{CONCLUSION}

UNE TRANSPOSITION INTERNE COMPLEXE

À l'issue de cette analyse, on peut tenter de schématiser le processus de transposition didactique qui a conduit à élaboration de la séquence (cf. figure 5 cidessous). 


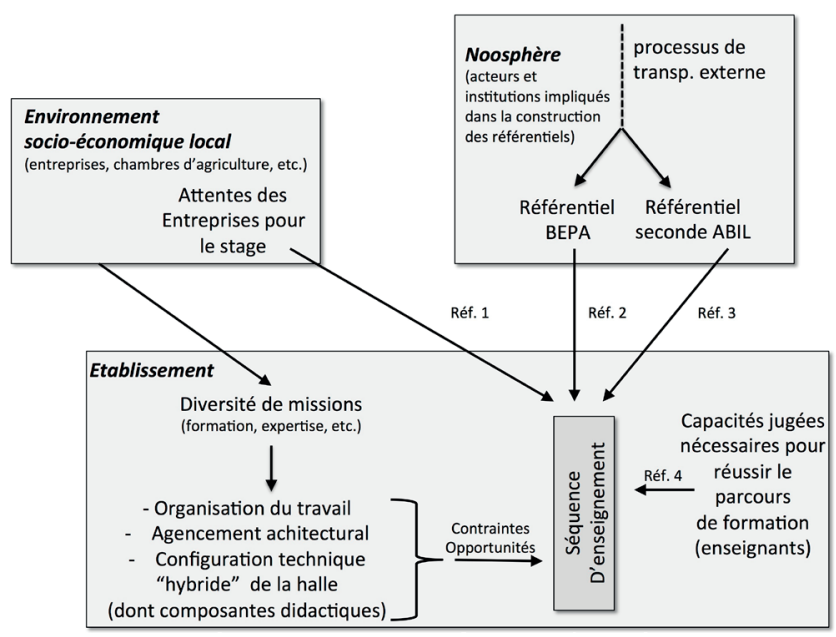

Figure 5 : le processus de transposition didactique interne dans le cas de la séquence étudiée.

Ce schéma met en évidence trois points remarquables. Tout d'abord, ce processus s'ancre dans plusieurs références de pratiques, situées à des niveaux différents : celles que décrivent les textes prescriptifs générés par le système national d'enseignement agricole, eux-mêmes issus d'un processus de transposition externe mené au niveau national en collaboration avec le monde professionnel ; celles des entreprises locales qui accueillent des jeunes en stage; et enfin des pratiques d'étude propres à l'établissement. On a là l'exemple d'une séquence qui cherche à répondre à plusieurs buts didactiques simultanément. On peut y voir le signe d'une complexité croissante des filières de formation qui cherchent à répondre à des objectifs de plus en plus nombreux. En réponse, il n'est pas illogique que les responsables de ces filières et leurs formateurs cherchent à trouver des dispositifs formatifs permettant (de leur point de vue) de répondre à plusieurs de ces objectifs tout en cherchant à réduire la complexité et la charge de travail (Veillard, 2015).

Le second point mis en lumière est que la séquence se sert de situations de formation préexistantes dans la halle (ateliers et laboratoires), mais que les enseignants concepteurs n'ont quasiment aucune prise sur celles-ci. Leur travail a donc consisté à analyser ces situations d'atelier, en identifier les opportunités et les contraintes, pour en proposer en amont une phase préparatoire, puis en aval une exploitation réflexive. Cela ressemble finalement beaucoup plus à ce qui est parfois fait en amont et en aval d'un stage en situation de travail qu'à l'idée que l'on peut se faire du travail d'ingénierie dans une école. Un indicateur supplémentaire de cette proximité avec les stages est que, comme dans ces derniers, il n'est pas possible d'anticiper les aléas qui ne manquent pas d'arriver dans les ateliers quotidiennement.

Enfin, le troisième et dernier point sur lequel nous permet d'insister ce schéma porte plus précisément sur l'organisation socio-technique autonome de la halle de génie alimentaire. Nous avons en effet montré en quoi cette organisation pèse assez largement dans le processus de transposition spécifique à cette séquence. C'est la raison pour laquelle il était nécessaire d'enquêter en amont sur cette organisation, en particulier sur les choix qui ont été faits pour aller dans le sens d'un certain aménagement didactique des lignes de fabrication tout en con- 
servant une logique productive forte. D'une certaine manière on peut considérer que ces choix d'aménagement des installations issus du passé et les tensions qu'ils génèrent ont largement contribué à la transposition didactique interne de la séquence étudiée.

\title{
Claire MASSON \\ doctorante UMR ICAR (Lyon 2) \\ DPF
}

(UBFC, Agrosup Dijon, Université Bourgogne Franche-Comté)

claire.masson@educagri.fr

Laurent VEILLARD

UMR ICAR

(CNRS, Université Lumière Lyon 2, ENS de Lyon)

laurent.veillard@univ-lyon2.fr

Paul OLRY

UP DPF

(UBFC, Agrosup Dijon)

paul.olry@educagri.fr

\begin{abstract}
This study is about the design and the implementation of a learning sequence in a secondary agricultural vocational college. We used the concept of internal didactic transposition for the analysis. We conducted an ethnographic investigation over a long period of time (3 years) and used a combination of different data production methods (observations, interviews, audio-video recordings, document analysis). Our results show the diversity of references which were at the origin of the transposition process under study. The transposition operations were complex, since they depended for a large part upon the socio-technical organization of the institution, which was the partial product of didactic reflections and choices, sometimes performed several years earlier.

Keywords : internal didactic transposition, school workshop, agricultural education,
\end{abstract} initial professional training.

\section{Bibliographie}

Arsac G., Chevallard Y., Martinand J. L. \& Tiberghien A. (1994) La transposition didactique à l'épreuve. Grenoble: La Pensée Sauvage.

Caillot M. (1996) «La théorie de la transposition didactique est-elle transposable ?»-in : C. Raisky \& M. Caillot (éds.) Au-delà des didactiques, le didactique. Débats autour de concepts fédérateurs (20-35). Bruxelles : de Boeck.

Chevallard Y. (1985/1991) La transposition didactique, du savoir savant au savoir enseigné. Grenoble : La Pensée Sauvage.

Durey A. (2002) «Impact de la CAO sur la définition des contenus d'enseignement en génie électronique »- in : J. Lebeaume et Y. Cartonnet (éds.) Séminaire de didactique des disciplines technologiques, 2001 2002. Bilan des recherches en didactique des disciplines technologiques (7-29). Cachan : Association Tour 123.

El Maaty N. (2007) «La scolarisation de l'apprentissage agricole en France : les fermes-écoles au service de l'agriculture et de son enseignement $\left(\mathrm{XIX}^{\mathrm{e}}\right.$ siècle-début $\mathrm{XX}^{\mathrm{e}}$ siècle) »-Ruralia 21 
en ligne, consulté le 07 octobre 2016. URL : http://ruralia.revues.org/1852

Gillet G., Veyrac H. \& Fraysse B. (2013) «Orchestrations instrumentales pour l'appropriation d'artefacts professionnels » - Travail et Apprentissage 11 (87-109).

Joshua S. (1997) « Le concept de Transposition Didactique peut-il étendre sa portée au-delà de la didactique des sciences et des mathématiques?»Skholé 6 (15-23).

Lipp A. \& Ria L. (2012) «La transmission des savoirs en formation professionnelle initiale: Analyse de l'activité d'enseignants en lycées agricoles »-Activités 9, 2 (71-87).

Marcus G. E. (1995) «Ethnography in/of the world system: the emergence of multi-sited ethnography » - Annual Review of Anthropology 24 (95117).

Martinand J.-L. (1986) Connaître et transformer la matière. Berne: Peter Lang.

Mercier A. (2002) «La transposition des objets d'enseignement et la définition de l'espace didactique, en mathématiques » - RPF 141 (135-171).

Métral J.-F. (2016) Entre situation de formation et situation professionnelle : les situations intermédiaires. Paris : L'Harmatan.

Olivier de Sardan J. P. (1995) «La politique du terrain » - Enquête 1 (71-109).

Pelpel P. \& Troger V. (1993) Histoire de l'enseignement technique. Paris : Hachette.

Perrenoud P. (1998) «La transposition didactique à partir de pratiques: des savoirs aux compétences »-Revue des Sciences de l'Éducation 24, 3 (487-514).

Perret J.-F. \& Perret-Clermont A.-N. (2001) Apprendre un métier dans un contexte de mutations technologiques. Fribourg : Éditions Universitaires.

Raisky C. (1996) «Doit-on en finir avec la transposition didactique ? Essai de contribution à une théorie didactique »- in : C. Raisky et M. Caillot (éds.) Au-delà des didactiques, le didactique. Débats autour de concepts fédérateurs (37-59). Bruxelles : de Boeck.

Veillard, L. (2015). De la division du travail de formation. Étude didactique de l'alternance en formation professionnelle initiale. HDR Université de Bourgogne.

Verret M. (1975) Le temps des études. Tome 1. Paris : Honoré Champion. 